\title{
Hollow-ion formation in microcapillaries
}

\author{
K. Tőkési, ${ }^{*}$ L. Wirtz, C. Lemell, and J. Burgdörfer \\ Institute for Theoretical Physics, Vienna University of Technology, Wiedner Hauptstrasse 8-10 /E-136, A-1040 Vienna, Austria
}

(Received 28 February 2001; published 17 September 2001)

\begin{abstract}
Transmission of highly charged ions through microcapillaries is studied theoretically by a classical trajectory simulation. The interaction of highly charged ions with the internal surface of the capillary is treated within the framework of dielectric-response theory. The simulation is based on the classical over-the-barrier model modified for open cylindrical surfaces. The multielectron evolution and relaxation is taken into account as a stochastic event sequence. We consider $\mathrm{N}^{6+}$ and $\mathrm{Ne}^{10+}$ with an energy of $2.1 \mathrm{keV} / \mathrm{amu}$ passing through a metallic microcapillary of Ni. We analyze the distance of closest approach, the angular distribution, and the distribution of the mean occupation numbers of $n$ shells of highly charged ions. We find the resulting charge state distribution of transmitted projectiles in good agreement with recent measurements. Implications for nanotube targets will be discussed.
\end{abstract}

DOI: 10.1103/PhysRevA.64.042902

PACS number(s): 34.50.Dy

\section{INTRODUCTION}

Collisions between highly charged ions (HCI) and solid surfaces are of growing interest and currently in the center of numerous experimental and theoretical works [1-22]. The main motivation of these efforts is the study of the basic interaction mechanisms between HCI and solid surfaces. Interest in these processes is stimulated, in part, by potential future technical applications, most notably, the possibility of selective desorption and nanostructuring of surfaces by HCIinduced potential sputtering [23]. From numerous experimental as well as theoretical studies, the following scenario of the HCI-surface interaction has emerged: When a highly charged ion approaches a solid surface, one or more electrons are resonantly captured at a characteristic distance into high Rydberg states of the projectile. As a result, a multiply excited Rydberg atom with inner-shell vacancies, a so-called hollow atom, is created. For metal surfaces charge transfer of the weakly bound conduction-band electrons into a highly charged ion sets in at large distances from the surface. In the literature, these hollow atoms are referred to as abovesurface hollow atoms or hollow atoms of the first generation (HA1). The classical over-the-barrier ( $\mathrm{COB})$ model $[3,7]$ is widely used to estimate the distance where the first resonant charge transfer can take place. For flat metal surfaces, the characteristic distance is approximately given by

$$
d_{c}=\sqrt{\frac{2 q}{W}}
$$

where $q$ is the charge of the ion and $W$ is the work function of the target. The capture takes place into the electronic shell of the projectile with a principal quantum number of the order of

\footnotetext{
*Permanent address: Institute of Nuclear Research of the Hungarian Academy of Sciences (ATOMKI), P.O. Box 51, H-4001 Debrecen, Hungary.
}

$$
n_{c} \approx \frac{q}{\sqrt{2 W}}\left(1+\frac{q-1 / 2}{\sqrt{8 q}}\right)^{-1 / 2} .
$$

The COB model also gives information about the interaction time $t_{I}$ for a $\mathrm{HCI}$ above the target surface that is determined by the image acceleration and the neutralization dynamics. As the ion further approaches the surface, a large number of electrons are captured into highly excited states. However, within the available time $t_{I}<10^{-13}$ seconds, complete relaxation to the neutral ground state is impossible, in other words, the lifetime of the HA1, save for collisional destruction at the surface, is much greater than $t_{I}$.

As the ion reaches the surface, the outer charge cloud is replaced by a much tighter one with the radius of the order of the bulk value of the metallic screening radius, forming a hollow atom of the second generation (HA2). A hollow ion/ atom can still exist temporarily at or below the surface [2427]. However, close collision with target atoms at the surface and bulk penetration provide a much more efficient pathway to complete relaxation. The HA1's formed above the surface are immediately and inevitably converted to HA2's at or below the surface. In turn, the information on the above-surface hollow atom is (to a large extent) erased, as its direct observation is limited to the time interval $t_{I}$. In particular, many properties of hollow atoms such as long-lived multiply excited resonances escape the observation. As a consequence of this scenario, HCI-surface collision experiments have two major difficulties in studying HA1's: (1) signals from HA2's are very often much stronger than those from HA1's as has already been observed by Meyer et al. [28,29], and (2) the survival times of HA1's are too short to study their intrinsic and quasistationary properties. Therefore, it is extremely difficult to directly observe and study the intrinsic properties of HA1's using flat surface targets.

One possible way to avoid the above-mentioned difficulties and preferentially prepare HA1's is to suppress production of HA2's using a special target shape in the experiments, namely stepped surfaces. In this case, the HA1's can be created without destructive close collision with the surface, and can be detected. One potential candidate for a stepped- 


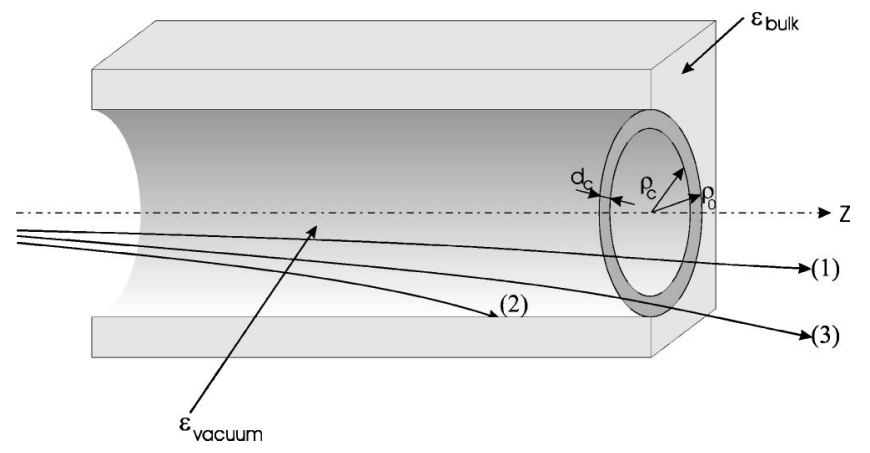

FIG. 1. Sketch of the ideal microcapillary with typical ion trajectories. $\rho_{c}$ is the critical capture radius and $d_{c}$ is the critical capture distance.

surface target is a microcapillary. Recently, interactions of HCI's with internal surfaces in microcapillaries have been introduced as an alternative technique to study above-surface processes [30-36]. The underlying idea is that by using capillary targets, the extraction of HA1's in vacuum and the observation of photons or Auger electrons emitted from them in flight becomes possible. Ions traveling approximately parallel to the capillary axis (Fig. 1) will be attracted by image forces toward the cylindrically shaped internal wall of the capillary [37-39]. When the distance of the ion to the wall reaches a critical value $\left|\rho_{0}-\rho\right| \leqslant\left|\rho_{0}-\rho_{c}\right|=d_{c}$, resonant charge transfer according to the $\mathrm{COB}$ model can take place $[3,7]$. While the dominant fraction of projectiles will either exit without experiencing charge transfer (trajectory type 1 , in Fig. 1) or undergo close collisions with the surface similar to conventional grazing surface collisions (trajectory type 2) leading to complete relaxation to the neutral ground state, trajectories of type 3 will undergo only large-distance "above-surface" collisions near the exit edge of the capillary and form HA1's. These ions can escape prior to hitting the wall and, hence, preserve the memory of the above-surface hollow-atom formation.

In this paper, the transmission of HCI through microcapillaries is studied by a classical trajectory simulation. The electronic dynamics of trajectory type 3 events is simulated within a Monte Carlo approach: electron capture, resonant ionization, radiative and nonradiative decay are followed as a random event-by-event sequence with rates taken from the COB model for capture and loss $[3,7]$ modified for the cylindrical geometry. The forces governing the ionic trajectory are, in turn, derived from the charge-state evolution determined by the stochastic electronic dynamics. We also consider deviations from the ideal geometry: We present simulations for the realistic capillary, when the "bumps," bottlenecks, and inhomogeneities are taken into account inside the microcapillary. Furthermore, we include fringe-field effects due to the capillary exit surface. In our simulation, we have used a metallic microcapillary of Ni. As projectiles, we use $\mathrm{N}^{6+}$, and $\mathrm{Ne}^{10+}$ with an energy of $2.1 \mathrm{keV} / \mathrm{amu}$. The interaction between the $\mathrm{HCI}$ and the capillary surface is taken into account by the classical image potential within the framework of dielectric response. The dielectric response of the capillary material is described by a plasmon-pole approximation in terms of a sum of Drude-type functions. We note, however, that the applicability of the dielectric response and, hence, of the linear-response theory to the present case of slow highly charged ions with $q / v \gg 1$ is $a$ priori not obvious. Its justification rests on the quasistatic limit of weak perturbations at large distances rather than on short interaction times as implied by $q / v<1$. Simple estimates [7] indicate that for distances $d>r_{s} \sqrt{q}$, nonlinear effects should still be weak where $r_{s}$ is the Wigner-Seitz radius. The distant ion-capillary surface interactions should therefore be well approximated by the dielectric response.

Results will be presented for the charge-state distribution, the distances of closest approach, the angular distributions for the transmitted HCI's, and for the distribution of the mean occupation numbers of $n$ shells for different charge states. Our calculated data will be compared with the available experimental data.

\section{THEORETICAL FRAMEWORK}

\section{A. Image potential of the infinite capillary}

We consider a slow ion with charge $q$ traveling through a microcapillary of radius $\rho_{0}$ with velocity $\vec{v} \quad(v \approx 0.1-0.5$ a.u.). The solid located outside the cylindrical opening (Fig. 1 ) is assumed to be characterized by an isotropic dielectric constant $\boldsymbol{\epsilon}(k, \omega)=\epsilon_{\text {bulk }}(k, \omega)$ which is a function of the frequency $\omega$ and wave-number $k$ of the electromagnetic disturbance. The electric potential induced by the traveling ion is determined from the Poisson equation

$$
\nabla^{2} \Phi=-4 \pi q \delta(\vec{r}-\vec{v} t) .
$$

This boundary-value problem can be conveniently solved using cylindrical coordinates. The potential inside the cylinder $\left(\rho<\rho_{0}\right)$ is given by [40-42]

$$
\begin{aligned}
\Phi^{<}(\rho, \phi, z)= & \frac{1}{2 \pi} \int d \omega \sum_{m} \int d k A_{m} \mathbf{e}^{i m \phi} \mathbf{e}^{i k z} \mathbf{I}_{\mathbf{m}}(k \rho) \mathbf{e}^{-i \omega t} \\
& +\frac{q}{\left|\vec{r}-\vec{r}_{1}\right|}
\end{aligned}
$$

and outside $\left(\rho>\rho_{0}\right)$ by

$$
\Phi^{>}(\rho, \phi, z)=\frac{1}{2 \pi} \int d \omega \sum_{m} \int d k B_{m} \mathbf{e}^{i m \phi} \mathbf{e}^{i k z} \mathbf{K}_{\mathbf{m}}(k \rho) \mathbf{e}^{-i \omega t},
$$

where the ion is located at $\vec{v} t=\vec{r}_{1}=\left(\rho_{1}, \phi_{1}, z_{1}\right), \mathbf{I}_{\mathbf{m}}$ and $\mathbf{K}_{\mathbf{m}}$ are the modified Bessel functions of order $m$ of the first and second kind, respectively. The first term in Eq. (4) is the induced potential and the second term is the electrostatic potential of the moving projectile, which can be expanded as [42]

$$
\begin{aligned}
\frac{q}{\left|\vec{r}-\vec{r}_{1}\right|}= & \frac{q}{\pi} \int d \omega \sum_{m} \int d k \mathbf{e}^{i m\left(\phi-\phi_{1}\right)} \mathbf{e}^{i k\left(z-z_{1}\right)} \\
& \times \mathbf{I}_{\mathbf{m}}\left(k \rho_{<}\right) \mathbf{K}_{\mathbf{m}}\left(k \rho_{>}\right) \mathbf{e}^{-i \omega t},
\end{aligned}
$$


where $\rho_{<}\left(\rho_{>}\right)$is the smaller (larger) of $\rho$ and $\rho_{1} . A_{m}$ and $B_{m}$ can be determined from the boundary conditions at the interface

$$
\begin{gathered}
\Phi^{<}\left(\rho=\rho_{0}\right)=\Phi^{>}\left(\rho=\rho_{0}\right), \\
\left.\frac{\partial \Phi^{<}}{\partial \rho}\right|_{\rho=\rho_{0}}=\left.\epsilon(k, \omega) \frac{\partial \Phi^{>}}{\partial \rho}\right|_{\rho=\rho_{0}} .
\end{gathered}
$$

The resulting image potential can be written as

$$
\begin{aligned}
\Phi(\rho, \phi, z)= & -\frac{q}{\pi} \int d \omega \sum_{m} \int d k \mathbf{e}^{i(k z-\omega t+m \phi)} \\
& \times \mathbf{I}_{\mathbf{m}}\left(k \rho_{1}\right) \chi_{\mathbf{m}}\left(k \rho_{0}\right) \mathbf{I}_{\mathbf{m}}(k \rho) \delta(\omega-k v),
\end{aligned}
$$

where

$$
\begin{aligned}
\chi_{\mathbf{m}}\left(k \rho_{0}\right)= & {[\boldsymbol{\epsilon}(k, \omega=k v)-1]\left[\boldsymbol{\epsilon}(k, k v) \frac{\mathbf{I}_{\mathbf{m}}\left(k \rho_{0}\right)}{\mathbf{K}_{\mathbf{m}}\left(k \rho_{0}\right)}\right.} \\
& \left.+\frac{k \rho_{0} \mathbf{I}_{\mathbf{m}-1}\left(k \rho_{0}\right)-m \mathbf{I}_{\mathbf{m}}\left(k \rho_{0}\right)}{k \rho_{0} \mathbf{K}_{\mathbf{m}-1}\left(k \rho_{0}\right)+m \mathbf{K}_{\mathbf{m}}\left(k \rho_{0}\right)}\right]^{-1}
\end{aligned}
$$

In the limit of an ideal conductor $(\epsilon \rightarrow \infty)$, Eq. (9) becomes

$$
\Phi(\rho, \phi, z)=\frac{q}{2} \Phi\left(\rho=\rho_{1}, \phi, 0\right)=-\frac{q^{2} \rho_{1}^{2}}{2 \rho_{0}\left(\rho_{0}^{2}-\rho_{1}^{2}\right)} .
$$

It should be noted that Eqs. (9) and (11) pertain to an infinite cylinder. For a Ni capillary, this simplified approximation was found [43] to describe the projectile motion overall quite accurately. We note that Eq. (9) can also be applied to interactions of charged particles with nanotubes [44].

\section{B. Potentials for the active electron inside the capillary}

The slow HCI approaching the solid surface causes a collective response of the electrons in the solid, resulting in an image potential at a large distance from the surface described by Eq. (9). This potential provides one contribution to the total potential an "active" electron involved in the transition feels. The properties of the surface are characterized by a conduction band with the work function $W$ and the Fermi energy $E_{F}$. As the ion approaches the surface, at the characteristic distance $\left(d_{c}\right)$, the potential barrier between the projectile and the metal surface dips below the Fermi level, and charge transfer becomes classically allowed. The potential barrier is formed by the superposition of the potential of the projectile, its image potential, and the image potential of the electron to be captured. The potential as a function of the radial electronic coordinate $\rho$ along the internuclear axis and cylindrical symmetric case is given by

$$
V\left(\rho, \rho_{1}\right)=V_{e p}\left(\rho, \rho_{1}\right)+V_{e p}^{I}\left(\rho, \rho_{1}\right)+V_{e}^{I}(\rho),
$$

where the first term in Eq. (12) is the direct interaction potential between the active electron and the projectile and the second and third terms are the effective interactions of the electron with the projectile image and the self image, respectively. Using in the following for simplicity the limit $\epsilon \rightarrow \infty$ [Eq. (11)] $V\left(\rho, \rho_{1}\right)$ becomes

$$
V\left(\rho, \rho_{1}\right)=-\frac{q}{\rho-\rho_{1}}+\frac{\rho_{0} q}{\rho_{0}^{2}-\rho \rho_{1}}-\frac{\rho_{0}}{2\left(\rho_{0}^{2}-\rho^{2}\right)} .
$$

We note, however, that our numerical simulations employ the full dielectric response [i.e., Eq. (9)]. the saddle point can be obtained from the implicit equation,

$$
\left.\frac{\partial V\left(\rho, \rho_{1}\right)}{\partial \rho}\right|_{\rho=\rho_{s}}=0
$$

and the saddle potential follows the form

$$
V_{s}=V\left(\rho_{s}, \rho_{1}\right) .
$$

According to the COB model, capture takes place when the saddle potential $\left(V_{s}\right)$ is below the energy level of the electron to be transferred. The critical distance between projectile and surface, where the first charge transfer takes place can be calculated form the following condition:

$$
V_{s}\left(\rho_{s}, \rho_{1}=\rho_{c}\right)=W .
$$

The COB model predicts the critical principal quantum number $n_{c}$ into which the first capture takes place as

$$
n_{c}=\left\{\max [n] \mid E_{n}\left(\rho_{c}\right) \leqslant V_{s}\right\},
$$

where the atomic energy level [3] is given by the imagepotential shifted hydrogenic value

$$
E_{n}(\rho) \approx \frac{q-0.5}{2\left(\rho_{0}-\rho\right)}-\frac{q^{2}}{2 n^{2}} .
$$

\section{Dielectric function}

The evaluation of Eq. (9) requires data for the dielectric constant $\epsilon(k, \omega)$ for the microcapillary material. Since detailed information about $\epsilon(k, \omega)$ exists only for a few solids and a rather restricted range of $\omega$ and $k$ values, we use experimental optical data [i.e., $\varepsilon(\omega)]$ for extrapolating into the $(k, \omega)$ plane. In the following two alternative strategies for the extrapolation will be discussed. In both cases, we employ a plasmon-pole approximation.

Ritchie and Howie [45] have proposed a method to extend the dielectric function to $k \neq 0$ as a sum of several terms of Drude-type dielectric functions assuming a single-pole dispersion relation. A Drude-type dielectric function can be written as

$$
\epsilon_{D}\left(k, \omega, \omega_{p}\right)=1+\frac{\omega_{p}^{2}}{\omega_{k}^{2}-\omega_{p}^{2}-\omega(\omega+i \Gamma)},
$$

where the momentum dependence is explicitly taken into account through the dispersion relation $\omega_{k}=\omega_{k}\left(k, \omega_{p}\right)$. At $k=0$ and, $\omega_{k}=\omega_{p}, \varepsilon_{D}$ becomes 


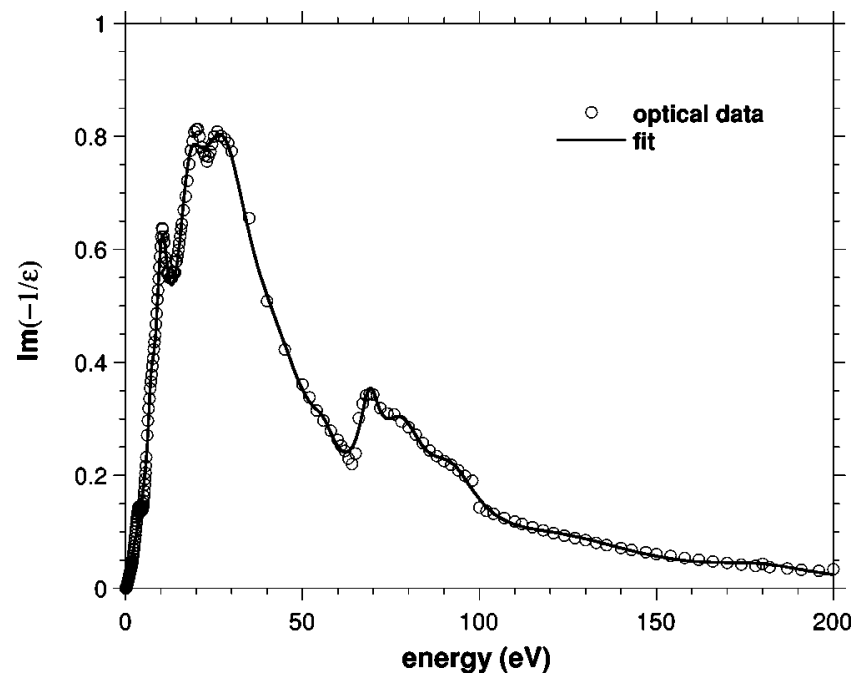

FIG. 2. Energy-loss function of Ni. Open circles, optical energyloss function derived from experimental data [48]; solid line, fit to the experimental results using a sum of Drude-type functions.

$$
\epsilon_{D}\left(\omega, \omega_{p}, \Gamma\right)=1-\frac{\omega_{p}^{2}}{\omega(\omega+i \Gamma)},
$$

with

$$
\omega_{p}=\sqrt{4 \pi n}
$$

where $\omega_{p}$ is the plasmon frequency and $n$ the electron density. $\Gamma$ denotes the full width at half maximum of the plasmon peak at frequency $\omega_{p}$. In this approximation, the energy-loss function, i.e., the imaginary part of $(-1 / \varepsilon)$ can be written as a sum over several Drude functions

$$
\operatorname{Im}\left\{-\frac{1}{\varepsilon(k, \omega)}\right\}=\sum_{j} \frac{A_{j} \Gamma_{j} \omega}{\left[\left\{\omega_{k}\left(k, \omega_{p j}\right)\right\}^{2}-\omega^{2}\right]^{2}+\left(\Gamma_{j} \omega\right)^{2}} .
$$

Following [45-47] the dielectric material is described in terms of several plasmonlike peaks. To evaluate the constants $A_{j}, \Gamma_{j}$, and $\omega_{p j}$, we fitted the optical data for Ni as compiled by Palik [48] by Eq. (22) at $k=0$. Equation (22) describes the influence of single-particle interactions for large momentum transfers and interactions with plasmons for small momentum transfers. Irrespective of the particular form of the dispersion relation discussed below, the resulting $\varepsilon(k, \omega)$ satisfies the generalized Thomas-Reiche-Kuhn sum rules [49].

Figure 2 shows the energy-loss function determined from experimental optical data and our fit to the experimental results using a sum of 13 Drude functions. The set of parameters determined by the fit of $\mathrm{Ni}$ data is shown in Table I.

In order to assess the dependence on the particular form of approximation for $\varepsilon(k, \omega)$, we also implemented an alternative extension of the dielectric function to $k \neq 0$ avoiding the tedious numerical procedure of parameter fitting. Penn [50] suggested a scheme, in which the summation in Eq. (22) over the finite number of Drude terms is replaced by an integration over the Drude dielectric function
TABLE I. Parameters used to fit the energy-loss data of [48] by the sum of Drude functions of Eq. (22).

\begin{tabular}{lrrr}
\hline \hline $\mathrm{i}$ & $A_{i}$ (a.u.) & $\omega_{p i}($ a.u. $)$ & $\Gamma_{i}$ (a.u.) \\
\hline 1 & 0.0000239 & 0.0735 & 0.132 \\
2 & 0.000272 & 0.162 & 0.126 \\
3 & 0.00167 & 0.269 & 0.0653 \\
4 & 0.0233 & 0.386 & 0.168 \\
5 & 0.0781 & 0.684 & 0.349 \\
6 & 0.514 & 1.075 & 0.813 \\
7 & 0.417 & 1.654 & 1.093 \\
8 & 0.0331 & 2.058 & 0.318 \\
9 & 0.0979 & 2.539 & 0.257 \\
10 & 0.289 & 2.880 & 0.602 \\
11 & 0.266 & 3.404 & 0.717 \\
12 & 0.757 & 4.662 & 2.432 \\
13 & 0.206 & 6.661 & 1.433 \\
\hline \hline
\end{tabular}

$$
\operatorname{Im}\left\{\frac{-1}{\varepsilon(k, \omega)}\right\}=\int_{0}^{\infty} d \omega_{p} G\left(\omega_{p}\right) \operatorname{Im}\left\{\frac{-1}{\varepsilon\left(k, \omega, \omega_{p}\right)}\right\},
$$

where the weight factors $A_{j}$ are replaced by the expansion coefficient $G\left(\omega_{p}\right)$ in the spectral density. If the damping constant is infinitesimally small, $\operatorname{Im}(-1 / \varepsilon)$ becomes a $\delta$ function

$$
\lim _{\Gamma \rightarrow 0^{+}} \operatorname{Im}\left\{\frac{-1}{\varepsilon\left(k, \omega, \omega_{p}\right)}\right\}=\frac{\pi}{2} \frac{\omega_{p}^{2}}{\omega_{k}} \delta\left(\omega-\omega_{k}\right) .
$$

Hence, using Eq. (24) in Eq. (23) for $k=0$ leads to

$$
G(\omega)=\frac{2}{\pi \omega} \operatorname{Im}\left\{\frac{-1}{\varepsilon(\omega)}\right\} .
$$

Due to the $\delta$ function, the extrapolation procedure from $\varepsilon(\omega)$ to $\epsilon(k, \omega)$ is greatly simplified. One finds

$$
\varepsilon(k, \omega)=\frac{\omega_{0}}{\omega} \varepsilon(\omega),
$$

where $\omega_{0}$ is the positive frequency solution of

$$
\omega_{k}\left(k, \omega_{0}\right)=\omega
$$

Therefore, Eq. (23) is an alternative version of Eq. (22) by summing over an infinite number of undamped Drude terms. Up to this point, neither in Eq. (22) nor in Eq. (26) have we specified the dispersion-relation $\omega_{k}\left(k, \omega_{p}\right)$. Certainly, our choice should yield the optical limit $\omega_{k}(k \rightarrow 0)=\omega_{p}$ as $k$ $\rightarrow 0$ and the free-particle limit $\omega_{k}=k^{2} / 2$ as $k \rightarrow \infty$, corresponding to the Bethe ridge. One popular choice of Eq. (27) is [51]

$$
\omega_{k}^{2}=\omega_{p}^{2}+\frac{1}{3} v_{F}^{2}\left(\omega_{p}\right) k^{2}+\left(k^{2} / 2\right)^{2}
$$

where $v_{F}$ is the Fermi velocity. 

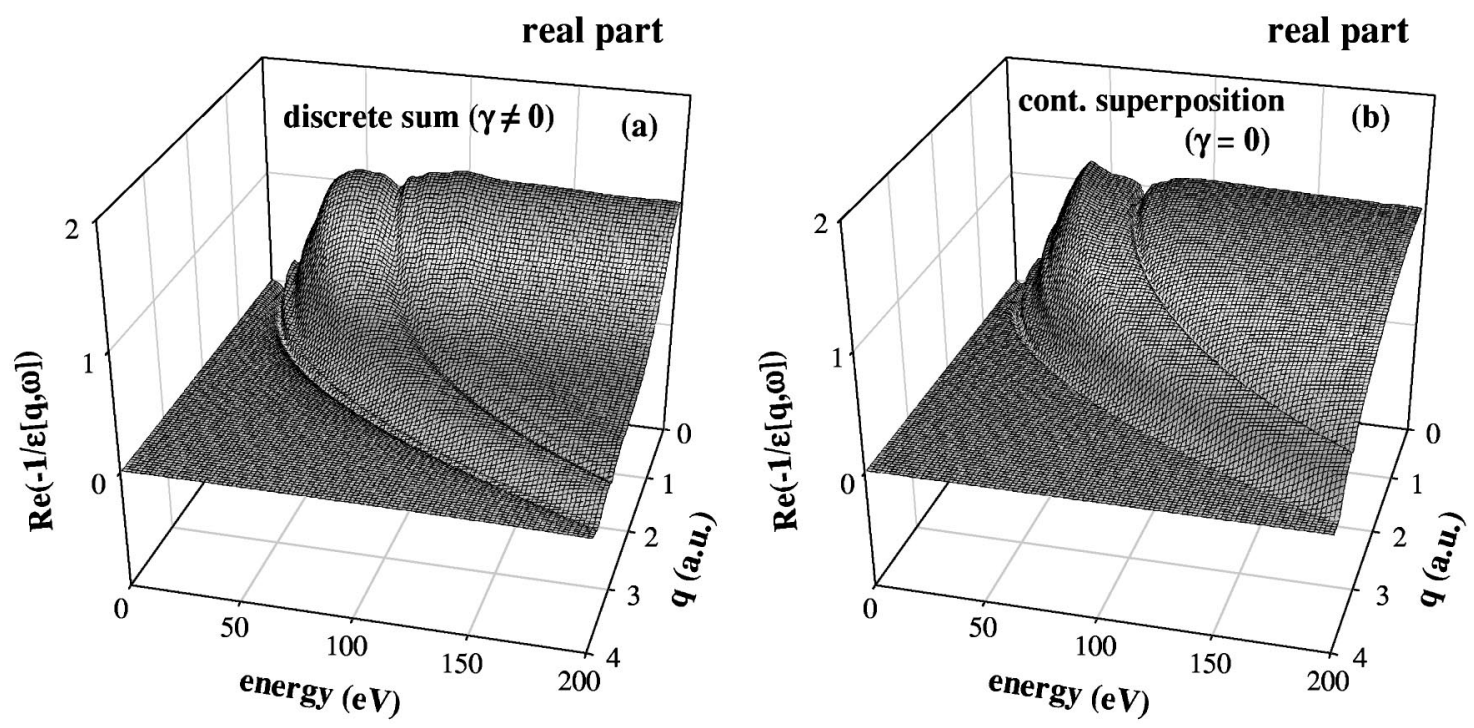

FIG. 3. Surface plot of the real part of the dielectric function for nickel (a) evaluated from Eq. (19) and (b) evaluated from Eq. (26).

Figures 3 and 4 show surface plots of the real and imaginary parts of the dielectric function of nickel as a function of momentum and frequency as given by Eq. (22) (discrete summation with finite damping constants) and Eq. (26) (continuous superposition with zero damping constants), respectively. In both figures, the Bethe ridge can be clearly seen. The resulting real and imaginary parts of the dielectric function are very similar using different approximations, indicating a weak dependence on the extrapolation procedure for $k \neq 0$. The simulations presented in the following are based on Eq. (22).

\section{Electric-field vector for ideal cylinder}

From the knowledge of the image potential and the dielectric function, the electric-field vector components can be calculated easily. The $\rho$ component is given by

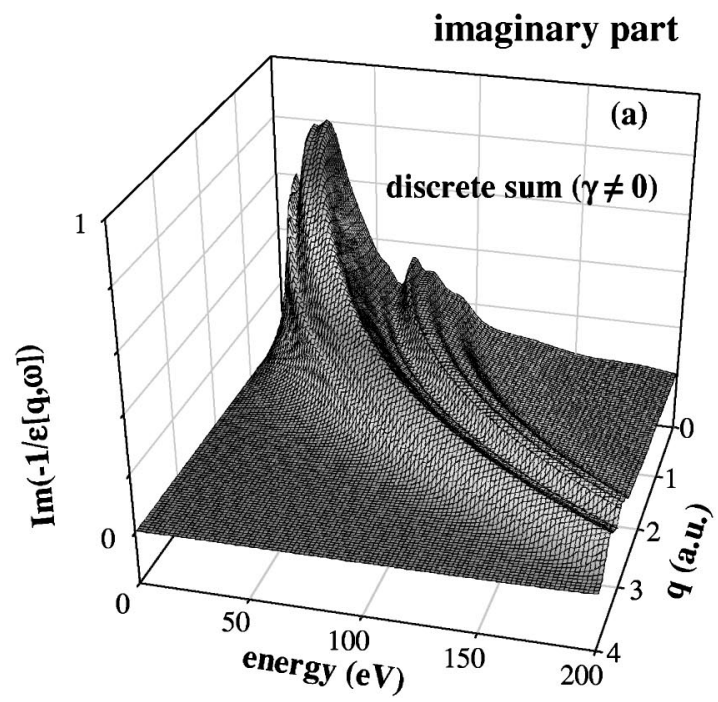

$$
\begin{gathered}
E_{\rho}=-\left.\frac{\partial \Phi_{i n d}}{\partial \rho}\right|_{\left(\vec{r}=\vec{r}_{1}\right)}, \\
E_{\rho}=\frac{q}{\pi} \int d \omega \sum_{m} \int d k \mathbf{I}_{\mathbf{m}}\left(k \rho_{1}\right) \operatorname{Re}\left[e^{i m \varphi} \chi_{m}\left(k \rho_{0}\right)\right] \\
\times\left[k \mathbf{I}_{\mathbf{m}-1}\left(k \rho_{1}\right) \frac{m}{\rho_{1}} \mathbf{I}_{\mathbf{m}}\left(k \rho_{1}\right)\right] \delta(\omega-k v) .
\end{gathered}
$$

In a similar way, we can get the $z$ component of the electric vector

$$
\begin{aligned}
E_{z}= & \frac{i q}{\pi} \int d \omega \sum_{m} \int d k k \mathbf{I}_{\mathbf{m}}^{2}\left(k \rho_{1}\right) \\
& \times \operatorname{Im}\left[e^{i m \varphi} \chi_{m}\left(k \rho_{0}\right)\right] \delta(\omega-k v) .
\end{aligned}
$$

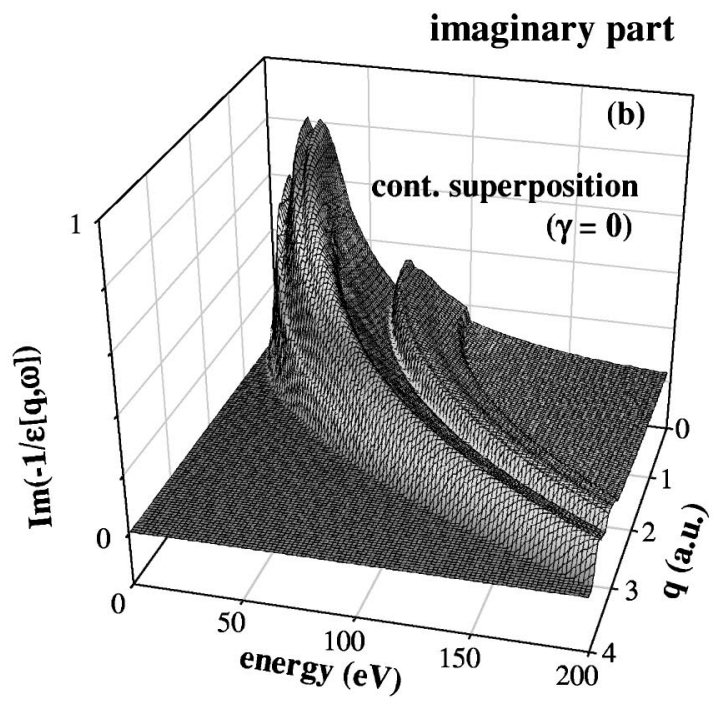

FIG. 4. Surface plot of the imaginary part of the dielectric function for nickel (a) evaluated from Eq. (19) and (b) evaluated from Eq. (26). 

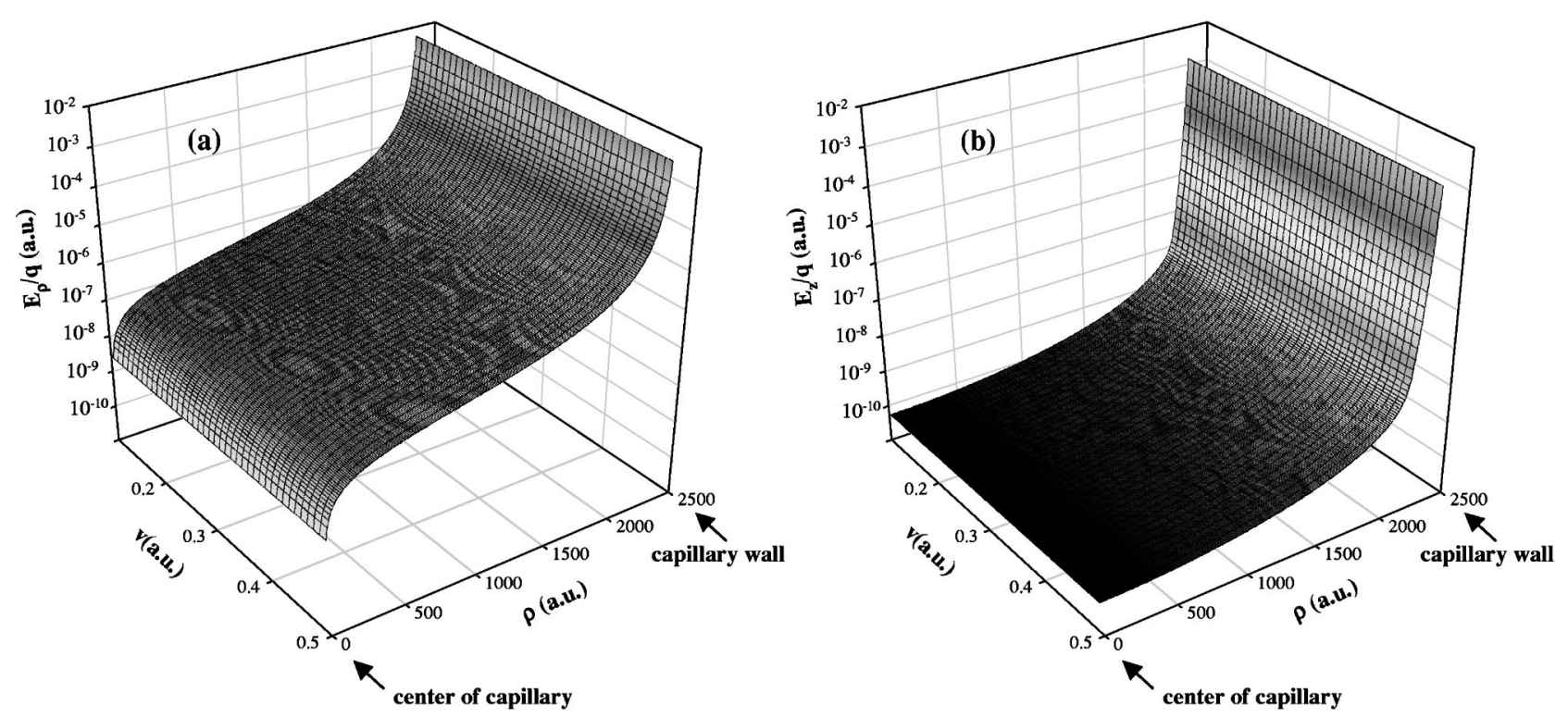

FIG. 5. Two-dimensional distribution of the electric field for an ideal cylinder as a function of the velocity of the charged particle and the cylindrical coordinates $\rho$ of the moving charge. (a) $E_{\rho}$; (b) $E_{z}$.

Figure 5 shows the $E_{\rho}$ and $E_{z}$ components of the electricfield vector as a function of the velocity of the projectile and as a function of the cylindrical coordinates $\rho$ of the moving charge. Both components are very small near the center of the capillary. However, the strength of the components strongly increases as the particle approaches the wall. The physical implications of the components of the field vector are as follows: $E_{\rho}$ governs, approximately, the image acceleration of the ion in the direction of the capillary wall, while $E_{z}$ is closely related to the stopping power.

\section{E. Deviation from ideal capillary geometry}

In our simulations, a Ni capillary with a nominal radius $\rho_{0}$ of $125 \mathrm{~nm}(\approx 2360$ a.u. $)$ and length $L=1.5 \mu \mathrm{m} \approx 28400$ a.u. was used. Since ensembles of experimentally available capillaries do not form ideal cylinders but have "bumps," bottlenecks, and inhomogeneities, these deviations from the ideal geometry are taken into account within a statistical ensemble. Figure 6 shows the sketch of a "model" for a "realistic" microcapillary surface. Deviations from the ideal capillary symmetry are simulated by allowing the cylindrical radius to vary randomly by $\pm 5 \%$ around its nominal value. The cylindrical walls are assumed to form terraces with

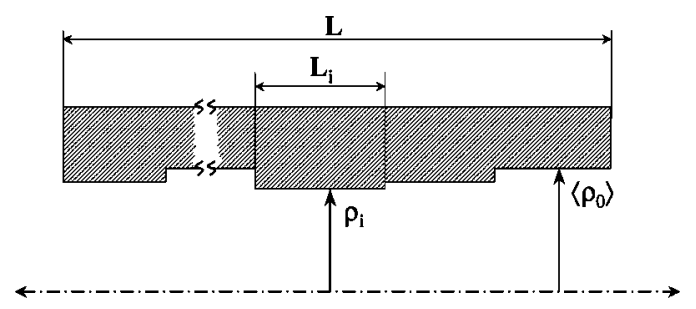

FIG. 6. Sketch of the realistic microcapillary surface. $L$ is the total length and $\left\langle\rho_{0}\right\rangle$ is the average radius of the capillary, respectively. lengths randomly distributed. The influence of the terrace length to the final charge-state distributions will be analyzed later in the paper.

\section{F. Fringe-field effects}

We take fringe-field effects near the edge of the exit surface of the capillary into account. For estimating corrections due to fringe fields, the cylindrically shaped surface is locally replaced by a planar surface (i.e., a Cartesian wedge) and thereby the potential for the infinitely extended cylinder is replaced near the exit plane and close to the edges by a wedge potential (see Fig. 7). This is justified since the relevant ensemble of trajectories we analyze in the following is near the exit surface close to within $\rho_{c} \approx 20$ a.u. of one of the walls (forming the wedge) while the opposite wall is at that point about $D=2 \rho_{0}=250 \mathrm{~nm}(\approx 4700$ a.u. $)$ apart. It is this large difference in scale $\left(\rho_{c} / D<10^{-2}\right)$ which allows the approximation by a flat surface. Note, however, that such an approximation does not hold in the interior of the capillary where the distances to the opposing walls can be comparable. We also note that for nanotubes where the diameter is

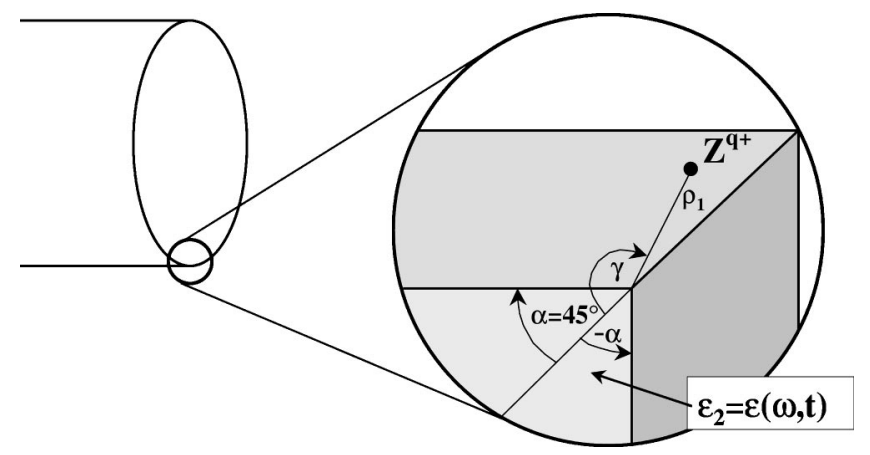

FIG. 7. Schematic view of the exit surface of the capillary with the wedge (shaded in gray) magnified. 


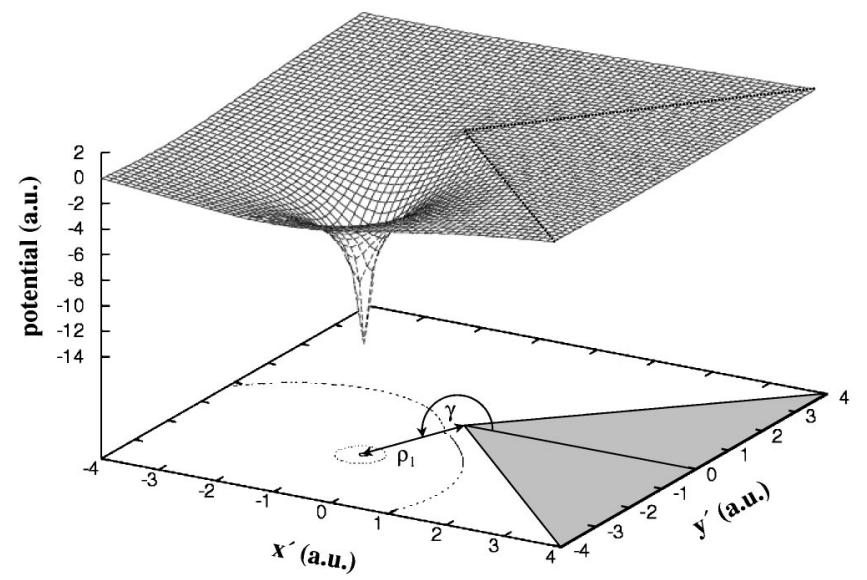

FIG. 8. Image potential surface for an ion $(q=1)$, near the wedge, in the rotated coordinate system. The $x^{\prime}$ axis coincides with the diagonal of the wedge.

on a nano- rather than a mesoscale, drastic changes are to be expected. Figure 7 displays the schematic view of the exit of the capillary with the model for the wedge. Extending the treatment of Ref. [52] for the electrostatic potential of a twodimensional wedge to the present case of the threedimensional wedge, the space was divided into three zones (Fig. 8). Let us denote the zone where the dielectric medium is located and which is bounded by the planes at $\theta=-\alpha$ and $\theta=\alpha$ by I, the zone bounded by the planes at $\theta=\alpha$ and $\theta$ $=\gamma$ by II, and the zone bounded by the planes at $\theta=\gamma$ and $\theta=-\alpha$ by III. The particle with charge $Z^{q+}$ is at $\theta=\gamma$ and $\rho=\rho_{1}$ (Fig. 7). The electrostatic potential in the different zones (Fig. 8) can be written as

$$
\begin{aligned}
V_{\mathrm{I}}(\rho, \phi, z)= & (1+\beta) \int d \omega \int d \nu \int d k \mathbf{e}^{i k z}\left(A e^{\nu \phi}+B e^{-\nu \phi}\right) \\
& \times \mathbf{I}_{i \nu}\left(k \rho_{1}\right) \mathbf{K}_{i \nu}(k \rho) \mathbf{e}^{-i \omega t}, \quad \text { if }-\alpha<\theta<\alpha, \quad 32 \\
V_{\mathrm{II}}(\rho, \phi, z)= & \int d \omega \int d \nu \int d k \mathbf{e}^{i k z}\left(C e^{\nu \phi}+D e^{-\nu \phi}\right) \\
& \times \mathbf{I}_{i \nu}\left(k \rho_{1}\right) \mathbf{K}_{i \nu}(k \rho) \mathbf{e}^{-i \omega t}, \quad \text { if } \alpha<\theta<\gamma, \\
V_{\mathrm{III}}(\rho, \phi, z)= & \int d \omega \int d \nu \int d k \mathbf{e}^{i k z}\left(E e^{\nu \phi}+F e^{-\nu \phi}\right) \\
& \times \mathbf{I}_{i \nu}\left(k \rho_{1}\right) \mathbf{K}_{i \nu}(k \rho) \mathbf{e}^{-i \omega t}, \quad \text { if } \gamma<\theta<2 \pi-\alpha,
\end{aligned}
$$

where

$$
\beta(k, \omega)=\frac{1-\varepsilon(k, \omega)}{1+\varepsilon(k, \omega)}
$$

The expressions, Eqs. (32)-(34), satisfy the Poisson equation. In order to satisfy the boundary conditions at the border of the zone $(\theta= \pm \alpha)$, the coefficients follow as

$$
\begin{gathered}
A=\frac{(\varepsilon+1) \sinh (\nu \pi) e^{-\nu(\gamma-\pi)}+(\varepsilon-1) \sinh [\nu(\pi-2 \alpha)] e^{\nu(\gamma-\pi)}}{(\varepsilon+1)^{2} \sinh ^{2}(\nu \pi)-(\varepsilon-1)^{2} \sinh ^{2}[\nu(\pi-2 \alpha)]}, \\
B=\frac{(\varepsilon+1) \sinh (\nu \pi) e^{\nu(\gamma-\pi)}+(\varepsilon-1) \sinh [\nu(\pi-2 \alpha)] e^{-\nu(\gamma-\pi)}}{(\varepsilon+1)^{2} \sinh ^{2}(\nu \pi)-(\varepsilon-1)^{2} \sinh ^{2}[\nu(\pi-2 \alpha)]}, \\
C=A+\beta B e^{-2 k \beta}, \\
D=\beta A e^{2 k \beta}+B, \\
E=\left(A+\beta B e^{2 k \beta}\right) e^{-2 k \pi}, \\
F=\left(A \beta e^{-2 k \beta}+B\right) e^{2 k \pi} .
\end{gathered}
$$

Figure 8 displays the electrostatic potential near the wedge surface for a charge $q=1$.

As the ion passes the wedge, its trajectory is modified by this potential. Due to the distortion by the fringe field, the asymptotic scattering angle and, thereby, the angular distribution of the HCI trajectories is shifted to larger scattering angles compared to a sudden switch off at the exit surface. This effect increases with higher charge states. The electricfield vector components are large only in the vicinity of the surface. However, in this region, the HCI is already almost neutralized. Therefore, the influence of the wedge potential on the ion trajectories should be minor. To illustrate the effect of fringe fields, we show in Fig. 9 the asymptotic angular distribution of $\mathrm{Ne}^{10+}$ trajectories with and without the wedge potential taken into account (for details of the trajectory calculation, see below). In order to highlight the effect, we use the frozen charge-state approximation, i.e., the charge state is kept fixed and the electron capture is neglected during the fly by. In the calculation labeled without wedge, the wedge potential is replaced by $V=0$ outside the capillary. From Fig. 9, it is obvious that the influence of the fringe field is very small. 


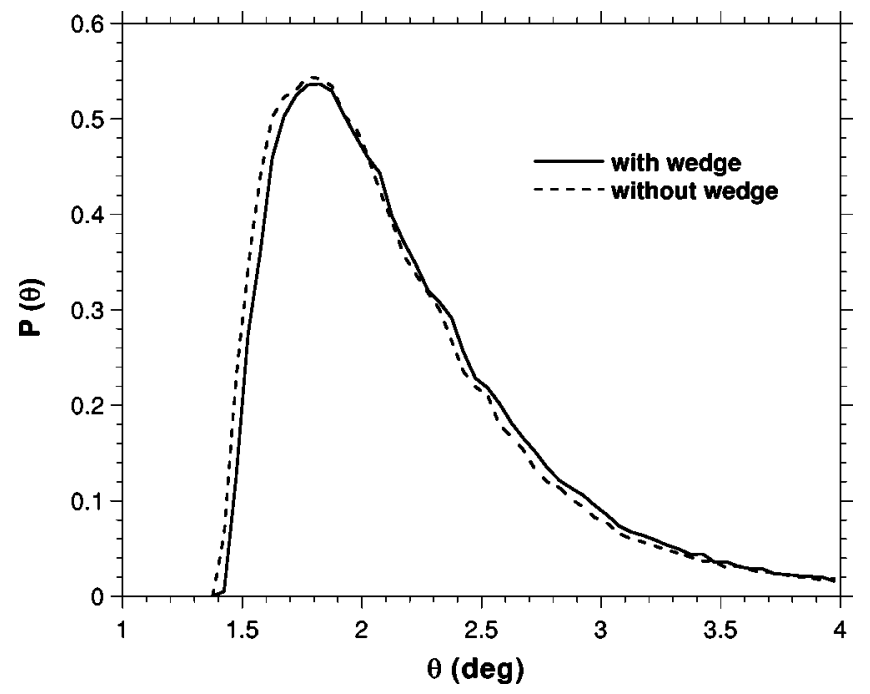

FIG. 9. Asymptotic angular distribution of $\mathrm{Ne}^{10+}$. Solid line, with correction due to the wedge effect; dashed line, without correction due to the wedge effect.

\section{G. State-changing processes}

As soon as the ion approaches the wall to within the critical distance $d_{c}$ for capture, the electronic and ionic dynamics have to be treated self consistently, since charge transfer influences the ionic trajectory. The electronic dynamics is simulated within a stochastic Monte-Carlo approach: electron capture, resonant ionization, radiative and nonradiative decay are followed as a random event-by-event sequence. Auger and radiative rates are taken from atomic structure codes $[25,53-58,62]$. Note that for any event, the influence of previous events along the history of one trajectory (e.g., ionic position, energy gain, charge state, shell occupation) is taken into account through a variation of relevant parameters that determine the probability for subsequent events. Within the independent-particle model, simultaneous multiple capture and loss is also included.

\section{Resonant loss}

An electron can be lost from the projectile when its orbit enters the region of the saddle. The frequency of collisions with the wall can be estimated from the period of Coulomb orbits

$$
\nu_{n}=\frac{q_{e f f}^{2}(n)}{2 \pi n^{3}},
$$

where $q_{\text {eff }}$ is the effective charge as seen in the $n$th shell of the hollow ion. In our numerical simulations, we determine the values of $q_{\text {eff }}$ according to the Slater screening rules [59]. The probability for hitting the opening of the potential wall in the saddle region is approximately given by

$$
P\left(E_{n}\right) \approx\left(1-\frac{\rho}{\rho_{c}^{E}\left(E_{n}\right)}\right),
$$

where in analogy to Eq. (16), $\rho_{c}^{E}\left(E_{n}\right)$ is the critical distance where a given energy transfer $\left(E_{n}\right)$ proceeds over the barrier and it can be calculated by

$$
V_{s}\left(\rho_{s}, \rho_{1}=\rho_{c}^{E}\right)=E_{n} .
$$

Loss into the solid can only take place when the energy of the level lies above the Fermi level. We calculate the loss probability per electron in the shell $n$ during the time interval $d t$ in the following form

$$
P_{n}^{\text {loss }}(\rho)=\Theta\left[W+E_{n}(\rho)\right] P\left(E_{n}\right) \nu_{n} d t,
$$

where $\Theta(x)$ is the Heaviside step function. The process of losing an electron is a Poissonian stochastic process. In the evolution of individual trajectories, we select the loss process with the help of uniform random number $R_{1} \in(0,1)$. Loss is assumed to take place when

$$
1-e^{-P_{n}^{l o s s}(\rho) f(n)}>R_{1},
$$

where $f(n)$ is the number of electrons in the $n$th shell. The same method applies to Auger processes and to radiative decays as well (see below).

\section{Ionization by promotion}

During the motion in the vicinity of the capillary surface, electron ionization due to promotion to the continuum can occur. To determine this rearrangement channel, we calculate the energy of the electron at a given distance [Eq. (18)]. If the energy balance $\Theta\left[E_{n}(\rho)\right]$ allow the promotion; the probability for this process to occur was set to 0.5 . Accordingly, the electron from the $n$ shell is promoted to the continuum state if

$$
\Theta\left[E_{n}(\rho)\right] \Theta\left(R_{2}-0.5\right)=1
$$

where $R_{2}$ is a uniform random number between 0 and 1 . If the ion is still within the critical capture distance instantaneous refilling by capture, however, into a different shell, will take place.

\section{Auger processes}

In our simulation, we take Auger ionization into account when two excited projectile electrons interact with each other. In our simulation, we neglect Auger deexcitation process in which a conduction- or valence-band electron fills a deeper lying level of the projectile ion, while an electron in an excited ion level is emitted carrying away the energy difference as kinetic energy, as well as the Auger capture channel, where two conduction- or valence-band electrons interact with each other and one of them is being transferred into a projectile level while the other is ejected. As it was shown by Hagstrum [60,61], these contributions are negligibly small compared to other relaxation channels at large distances from the surface.

For the determination of the Auger rate, we employ atomic structure calculation based on the Cowan code [62]. Following the procedure used in $[3,7]$ the rate for intra- 
atomic Auger process for two equivalent electrons in shell $n_{i}$ is approximated by

$$
\Gamma_{n_{i} ; n_{f}}=\frac{5.06 \times 10^{-3}}{\left(n_{i}-n_{f}\right)^{3.46}}
$$

The suppression of Auger decay due to partial population in the final shell $n_{f}$ can be taken into account by an empirical reduction factor [63]

$$
R\left(n_{f}\right)=1+1.5 \times f\left(n_{f}\right) .
$$

We use an additional correction factor, $\left(n_{i}^{\prime} / n_{i}\right)^{3}$ for electron pairs $\left(n_{i}, n_{i}^{\prime} ; n_{f}\right)$ which are not in the same shell (intershell) before the Auger decay. Finally, the Auger rate can be written in the following form:

$$
\Gamma_{n_{i}, n_{i}^{\prime} ; n_{f}}=\Gamma_{n_{i} ; n_{f}}\left(n_{i}^{\prime} / n_{i}\right)^{3} / R\left(n_{f}\right)
$$

The Auger process takes place if the energy balance for the given shells is fulfilled. We calculate the Auger probabilities at the time interval $d t$

$$
\begin{aligned}
P^{\text {Auger }}(\rho)= & \Theta\left[E_{n_{i}}(\rho)-E_{n_{f}}(\rho)\right. \\
& \left.-\left|E_{n_{i}^{\prime}}(\rho)\right|\right] \Gamma_{n_{i}, n_{i}^{\prime} ; n_{f}} f\left(n_{i}\right) f\left(n_{i}^{\prime}\right) d t .
\end{aligned}
$$

The Auger process takes place whenever

$$
1-e^{-P^{\text {Auger }}(\rho)}>R_{3},
$$

where $R_{3}$ is a uniformly distributed random number.

\section{Radiative decay, photon production}

In the radiative transition, a fraction of the potential energy of the hollow atom or ion is carried away by a photon. Radiative rates increase rapidly with the nuclear charge as $\sim Z^{4}$ for hydrogenic ions, whereas Auger rates depend only weakly on $Z$. Typical intra-atomic radiative probabilities are given by [64]

$$
P_{r} \simeq 4 \times 10^{-7} Z^{4} / n^{4.5} d t .
$$

In our trajectory calculations, the radiative decay takes place when

$$
1-e^{-P_{r} f(n)}>R_{4}
$$

where $R_{4}$ is a uniform random number between 0 and 1 . In our present calculations, inside the capillary, the effect of this relaxation channel for the ion trajectories is negligibly small. However, it becomes dominant at large distances from the exit surface for singly excited states relaxing to the final ground state.

\section{H. Classical equation of motion}

Using Eqs. (30) and (31) to calculate forces, ionic trajectories can be determined using the Euler-Lagrange equation

$$
\begin{gathered}
\frac{d}{d t}\left(\frac{\partial L}{\partial \dot{q}}\right)=\frac{\partial L}{\partial q}, \\
\ddot{\rho}-\rho \ddot{\varphi}=-\frac{1}{M} \frac{\partial \Phi_{\text {ind }}(\rho, \varphi, z)}{\partial \rho}, \\
\rho \ddot{\varphi}+2 \dot{\rho} \dot{\varphi}=-\frac{1}{M \rho} \frac{\partial \Phi_{\text {ind }}(\rho, \varphi, z)}{\partial \varphi}, \\
\ddot{z}=-\frac{1}{M} \frac{\partial \Phi_{\text {ind }}(\rho, \varphi, z)}{\partial z} .
\end{gathered}
$$

Here, $M$ is the mass of the HCI. Equations (56) and (58) were integrated using a standard fourth order Runge-Kutta method. The calculation of the ion trajectory is divided into three separate regions: (1) inside the capillary; (2) in the fringe-field region at the end of the capillary; and (3) the asymptotic region. From the critical distance where the resonant capture starts, the deexcitation channels are also taken into account using the Monte Carlo technique outlined above. When the ion is within the critical capture radius, the ion charge state does not change due to the deexcitation but only the electronic configuration of the ion will change. The underlying assumption is that a prompt recapture process takes place when the ionization stage is increased as a result of the relaxation. This is equivalent to the "staircase" model [65]. Outside the critical capture radius, however, the ion charge state is changed during the deexcitation accompanied by electron emission. It is important to realize that the charge-state distribution of the ensemble reaches its asymptotic stable limit only after the ion is about $100 \mu \mathrm{m}$, i.e., almost macroscopic distances, downstream from the exit surface when the Auger relaxation is complete. This is in sharp contrast to conventional specular reflection surface scattering processes where, as a result of close collisions, the final charge state is reached on a receding branch of the trajectory within a few $\AA$ above the surface. The long relaxation time toward the asymptotic charge state distribution $(\sim 0.1 \mathrm{~ns})$ is due to a sequence of comparatively slow Auger processes involving asymmetric configurations $\left(n l, n^{\prime} l^{\prime}\right)$ with $n \gg n^{\prime}$.

\section{RESULTS AND DISCUSSION}

The Ni surface is characterized by its work function $W$ $=5.2 \mathrm{eV}$. In order to study the hollow-atom (more precisely, hollow-ion) formation in microcapillary transmission, we have performed a classical trajectory simulation with an ensemble of $5 \times 10^{7}$ primary trajectories. The spatial distribution of the incident ensemble is uniform across the opening of the capillary cylinder.

\section{A. Coupling between ionic and electronic dynamics}

The influence of the charge-transfer process on trajectories passing by the exit surface is illustrated in Fig. 10 where we compare trajectories with frozen charge states as used in a preliminary investigation [43] with a self-consistent solu- 


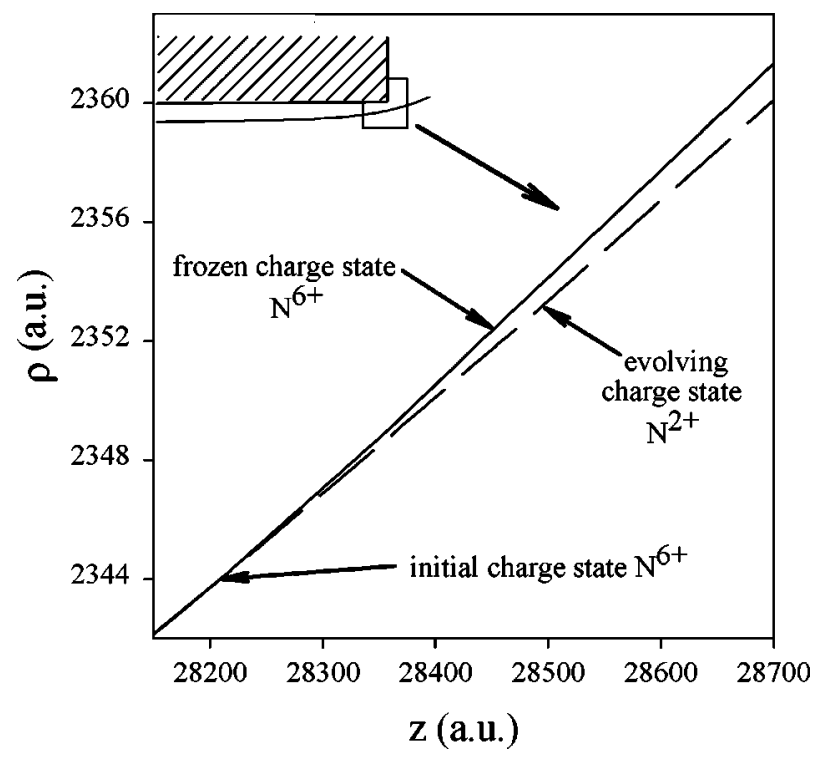

FIG. 10. Comparison between ionic trajectories of incident $\mathrm{N}^{6+}$ with frozen charge state $q=6$ and dynamically evolving charge state reaching $q=2$.

tion in which the trajectory is influenced by charge-state fluctuations. For the same incident charge state, the scattering angle for the frozen charge state is generally larger than for the case of dynamically evolving projectile charges due to the larger image acceleration in absence of the neutralization process. For the same reason, the dynamical evolution of the charge state reduces the number of close fly-by events and increases the average distance of closest approach to the exit edge.

\section{B. Time evolution of projectile charge state}

We analyze the charge-state distribution of outgoing $\mathrm{N}^{q+}$ ions for which experimental data are available [35]. To simulate the spread of the incident beam, we use a Gaussian angular distribution of $\theta_{i}$ with a full width at half maximum (FWHM) of 2 degrees, which is the estimated experimental value [66]. Extensions to more highly charged ions are straightforward and yield qualitatively similar results. In Fig. 11 , we compare the experimentally obtained final chargestate distribution of incident $2.1 \mathrm{keV} / \mathrm{amu} \mathrm{N}^{6+}$ ions transmitted through a Ni microcapillary [35] with our simulations. The overall agreement with the experimental data (open circles in Fig. 11) is remarkably good when we take into account Auger processes (solid circles). The data have been calculated assuming an average terrace length of 700 a.u. The dependence on the terrace length will be discussed below. The point to be noted is that only about $15 \%$ of the incident beam contributes to the transmission through the capillary while the remainder hits the entrance surface, undergoes close collisions with the walls, and is stopped in the capillary material near terraces. Within the subensemble of exiting ions, the incident charge state strongly dominates $(\sim 99 \%)$. The ratio of the incident charge-state fraction to all other charge states is directly related to the ratio of the entire capillary cross section of the cylinder to the area subtended

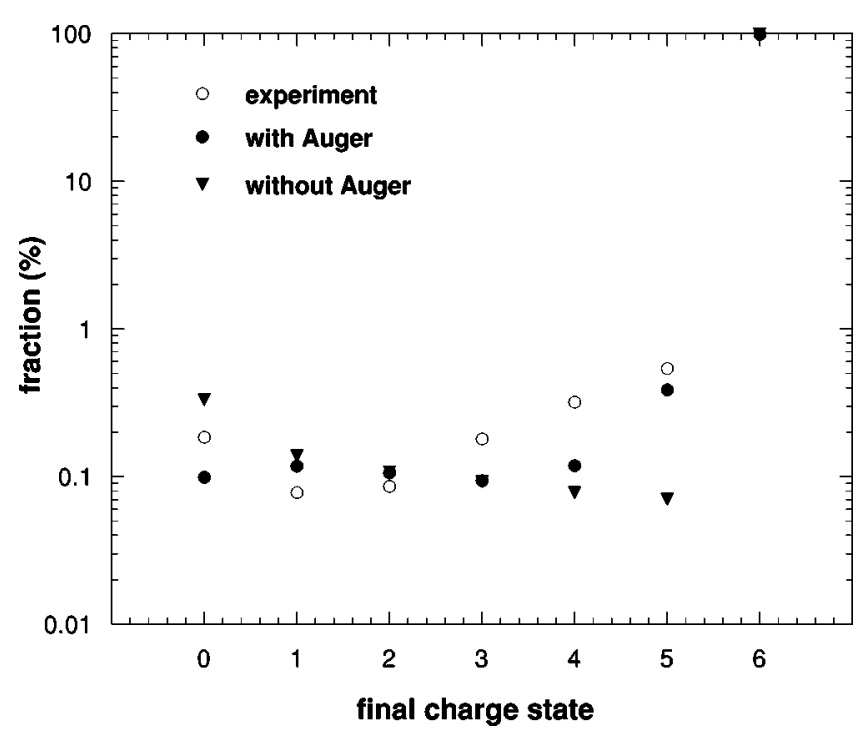

FIG. 11. Final charge state distribution of incident $2.1 \mathrm{keV} / \mathrm{amu}$ $\mathrm{N}^{6+}$ ions transmitted through a $\mathrm{Ni}$ microcapillary. Open circles, experiment [35]; solid circles, simulation with state changing processes taken into account; solid triangles, simulation without state changing processes taken into account.

by a ring of thickness $d_{c}$ (Fig. 1) and therefore provides a direct test for the distance of first capture. The present simulation gives overall good agreement for all ionic charge states on an absolute scale. The fairly flat and uniform distribution over all other charge states $(q=0,1,2,3,4,5)$ is a unique feature of distant collisions with the internal microcapillary surface, not previously observable in ion-atom, ionsolid, and grazing ion-surface collisions. It should be noted that only the fraction of neutrals $(q=0)$ in the experiment may contain a sizeable contribution from projectiles undergoing close collisions near the exit surface (trajectories type 2 in Fig. 1) which exit completely relaxed in the neutral ground state. For this charge state, the significance of the comparison between experiment and our simulation is a priori not clear. All the more surprising and probably fortuitous is the close agreement even for this charge state. The chargestate distribution for $q=1,2,3,4,5,6$ provides, in addition, detailed insight into the complex multielectron transfer process above the surface. As the final charge-state distribution depends also on a multitude of Auger relaxation processes, the distribution of electrons among $n$ shells of the hollow atom also appears to be predicted reasonably well.

As already mentioned, the neutral fraction of the transmitted particles can be contaminated by contributions from "hard" grazing collisions inside the capillary. These specularly reflected trajectories (SR) should also lead to neutral projectiles. The contribution of the specularly reflected ions to the neutral fraction is shown in Fig. 12. Here, we compare the fraction of neutral projectiles due to SR ion trajectories with the neutral fraction due to distant collisions as a function of the average terrace length. In each case, a uniform fluctuation in terrace length around the average value of $\pm 50 \%$ is assumed. In all cases, SR trajectories dominate.

Figure 13 shows the charge-state distributions for the shortest average terrace length from Fig. 12 simulating a 


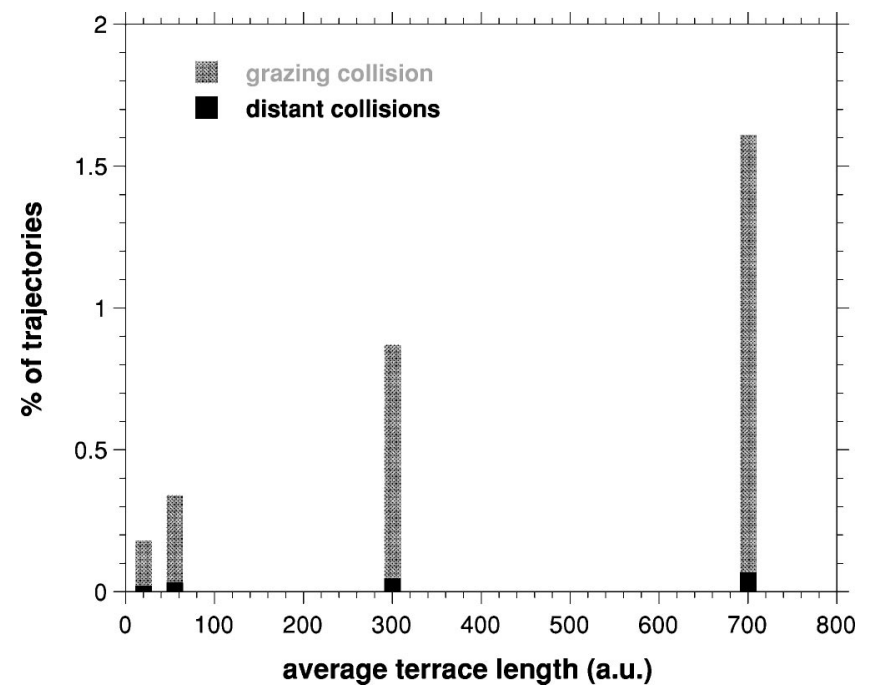

FIG. 12. Neutral fraction of the projectile beam exiting the capillary. Black columns, neutral fraction without hitting the capillary wall; gray columns, fraction of specularly reflected trajectories undergoing a close collision. Range of terrace length: (a) 10-30 a.u., (b) 10-100 a.u., (c) 100-500 a.u., (d) 400-1000 a.u.

rugged surface. With decreasing terrace length the neutral fraction of the transmitted projectiles due to the distant collisions decreases since the effective interaction time for both charge transfer and relaxation is reduced. We find improved agreement between the simulation of distant collisions (trajectories type 3) with experimental data except for $q=0$. This is precisely what we expected, since for neutrals the contribution due to grazing collisions dominate (Fig. 12). Including the latter for $q=0$, also shown in Fig. 13, leads to agreement for all charge states.

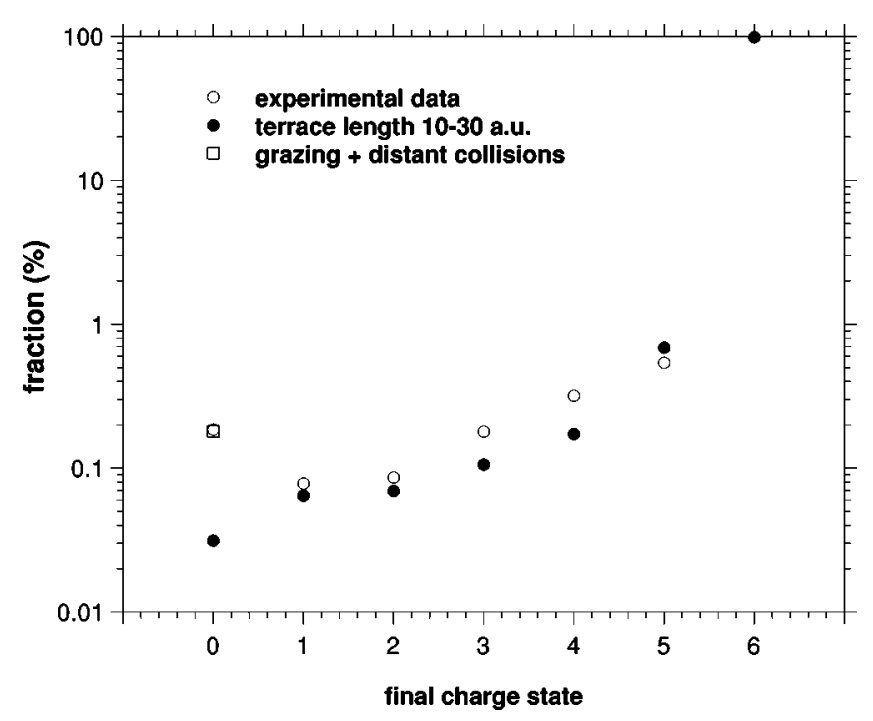

FIG. 13. Final charge state distribution of incident $2.1 \mathrm{keV} / \mathrm{amu}$ $\mathrm{N}^{6+}$ ions transmitted through a Ni microcapillary. Open circles, experiment [35]; solid circles, simulation of distant collision [trajectories (3) of Fig. 1] with average terrace length of 20 a.u.; open squares, simulation, for $q=0$ the grazing collision contribution [trajectories (2) of Fig. 1] is included.

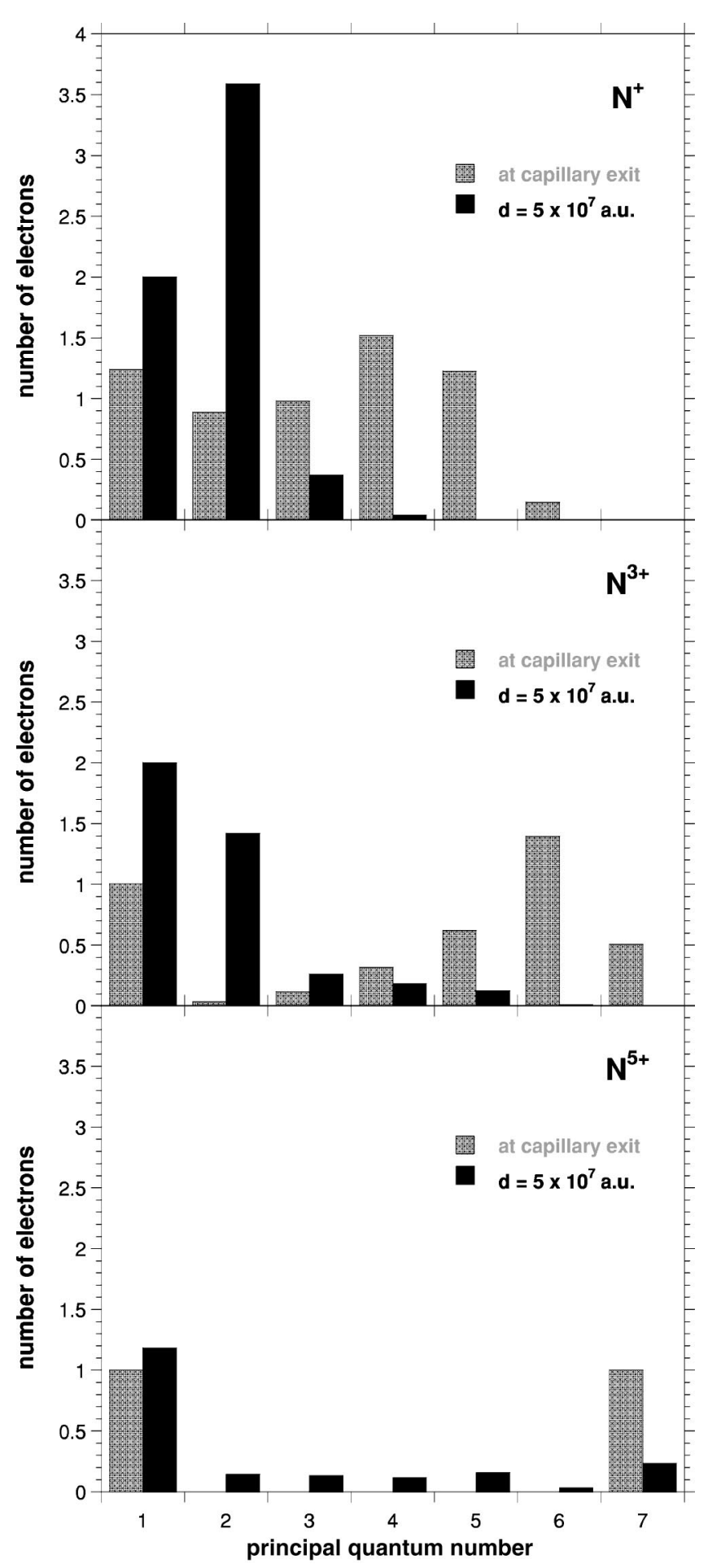

FIG. 14. Mean occupation numbers of $n$ shells for $\mathrm{N}^{6+}$ on nickel at different distances from the surface, light gray shadow: at the exit surface of the capillary, dark gray shadow: at mesoscopic distance $\approx 5 \times 10^{7}$ a.u. (after Auger relaxation, but with incomplete radiative cascade).

As the ion approaches the surface, highly excited states are resonantly populated. Deexcitation proceeds continuously via resonant ionization, Auger processes, and radiative decay. This results in a continuous shift of the population to lower $n$ shells. Although these rearrangement processes are 

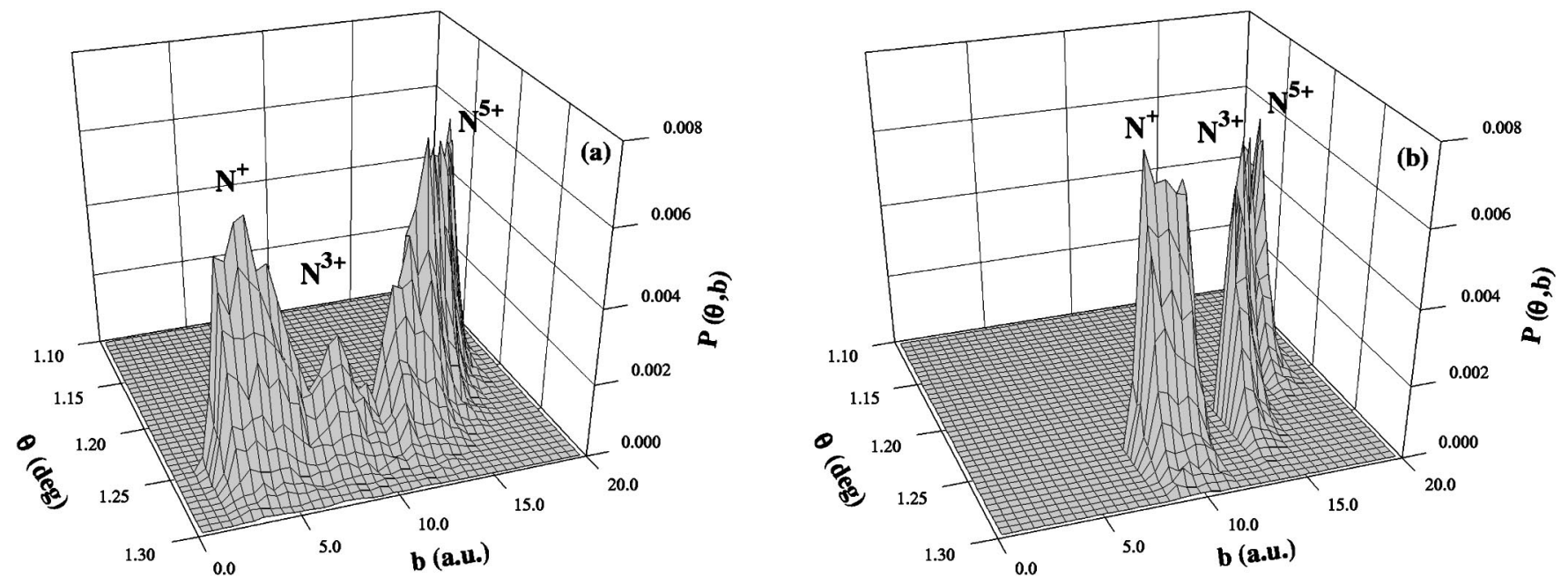

FIG. 15. Two-dimensional $P^{q+}(\theta, b)$ distribution of scattering angle $\theta$ and distance of closest approach $b$ for $\mathrm{N}^{6+}$ on nickel. (a) at macroscopic distance (after Auger relaxation) (b) at the exit surface of the capillary.

quite fast, the interaction time is far too short to lead to a complete deexcitation of the incoming projectiles. This is often referred to as the bottleneck problem. As a consequence, ions leave the capillary in highly excited states. Figure 14 shows the mean occupation numbers of $n$ shells for different projectile charge states. The snapshots were taken at two representative distances from the exit surface, outside the critical capture radius, and at large distances $\left(5 \times 10^{7}\right.$ a.u.) from the surface when all possible Auger processes, but not all radiative processes, have taken place. Ions in low charge states $(q=0,1,2)$ have lower $n$ shells strongly populated. Note that these charge states are correlated with small impact-parameter collisions with the capillary wall. We also note that these ion trajectories have the longest path lengths in the region where charge transfer from the surface is classically allowed. When the ion leaves this region, its charge will increase due to deexcitation via Auger decay into lower lying $n$ shells. As can be seen in Fig. 14, projectiles leaving the capillary in intermediate charge states $(q=3)$ form the well-known "hollow atoms" (HA1) with empty inner and partially filled outer shells. Deexcitation of these intermediate states can be investigated at large distances from the surface using Auger and photon spectroscopy increasing the asymptotic charge state fraction of $q=5$.

\section{Angular differential distributions}

From our simulations, we can also extract the angular $(\theta)$ distribution as well as the distance of closest approach $(b)$ distribution relative to the nearest exit edge (Fig. 15). To the extent that the $\theta$ and $b$ distributions are correlated, measurements of the charge-state distributions in coincidence with the scattering angle would provide the means to resolve subsequent stages of the hollow-atom formation above the surface. To demonstrate the angular separation, the angular divergence of the incident beam has been reduced in our simulation to a FWHM of $0.2^{\circ}$ (which is within experimental reach). Note that for higher incident charge states, the angular separation can be clearly seen even with much less stringent requirements on the initial beam divergence. Figure 15 displays the surface plot of the two-dimensional distributions $P^{q+}(\theta, b)$ for $q=1,3,5$ for $\mathrm{N}^{6+}$ on nickel, where panel (a) represents the asymptotic observable distributions, while (b) gives the distribution in close proximity to the exit surface. Similar to grazing incidence surface collisions $[6,65]$ the image acceleration of the HCI toward the wall manifests itself as a pronounced shift in the angular distribution towards larger scattering angles. We, indeed, observe a bananashaped $\theta-b$ correlation pattern with a very well-localized angular distribution. It is noteworthy that the angular distribution for each charge state is much narrower (FWHM $\leqslant 0.05^{\circ}$ ) than the initial angular divergence of the beam $\left(\mathrm{FWHM}=0.2^{\circ}\right)$. This is a direct consequence of the intrinsic high selectivity among all initial conditions of those that lead to transmission to the microcapillary accompanied by the charge exchange of a given charge state. Low charge states are strongly correlated with closer fly by's (small $b$ 's) and larger scattering angles $\left(\theta \simeq 1.25^{\circ}\right)$ while the effective single-capture channel $(q=5)$ extends from the threshold angle $\theta_{c}=1.13^{\circ}$ for single capture to larger angles. Notice that below $\theta_{c}$, no capture takes place since such angles would correspond to $b>d_{c}$. The comparatively broad angular distribution for the charge state $q=5$ is not due to a wider angular range for single capture in distant fly-by events but is due to the Auger decay of hollow atoms of (initially) lower charge states. To illustrate this effect, we show in Fig. 15(b) the $\theta-b$ correlation pattern for the same charge states but during flight as the ions pass through the exit surface. The evolution of this distribution to the one in Fig. 15(a) is exclusively due to the Auger cascade while the angular and the distances of closest-approach distribution pattern is already essentially frozen out. This simulation suggests that different stages of the hollow-atom (or -ion) formation should be directly accessible in future experiments provided that sufficient angular resolution can be achieved. Use of higher charge states would lessen the requirement on the angular resolution and facilitate the observation of the correlation pattern. 


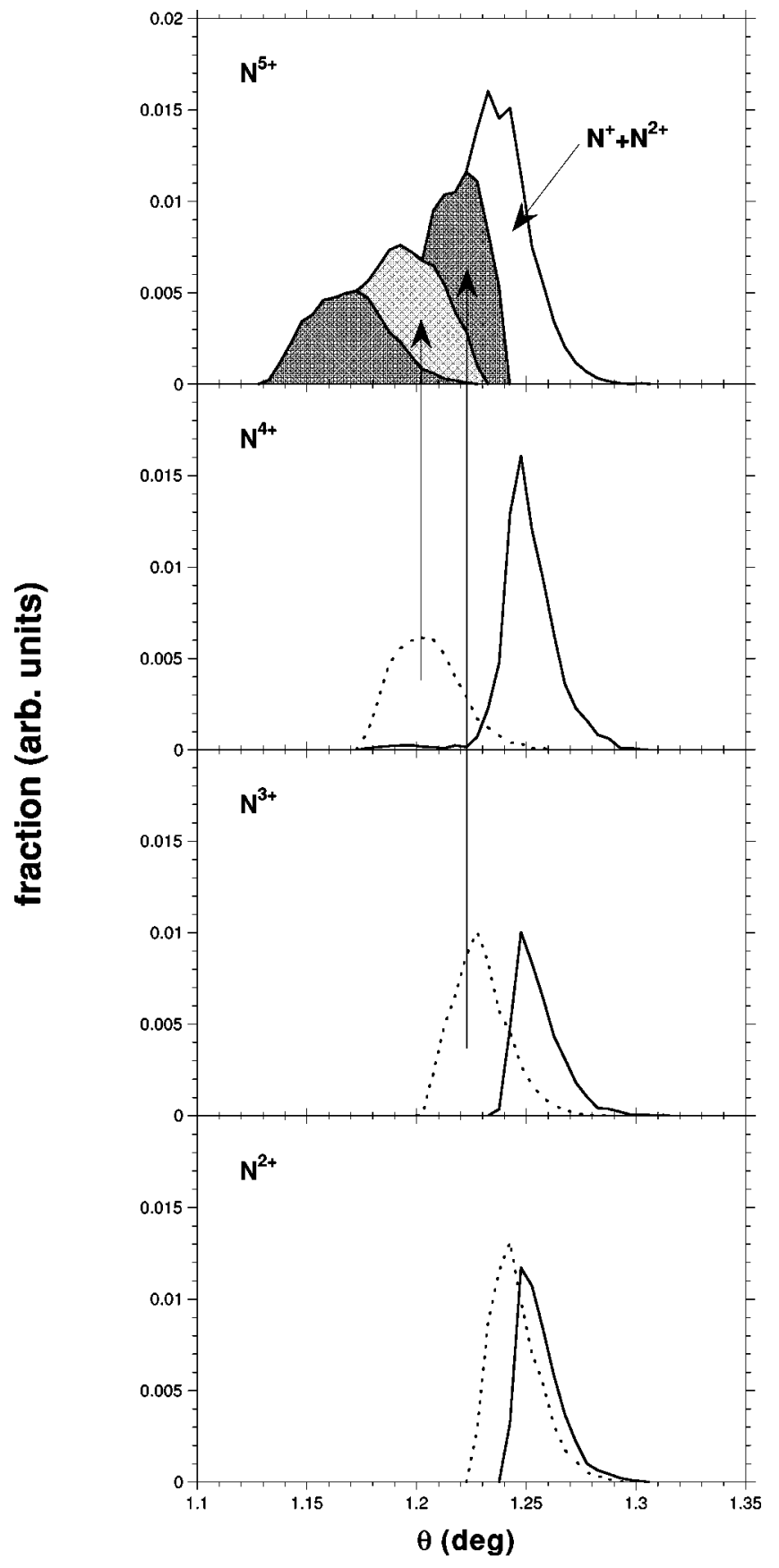

FIG. 16. Angular distributions for HCI's for different charge states. Solid lines, at $5 \times 10^{7}$ a.u. distance from the surface (after Auger relaxation); dashed lines, at the exit surface of the capillary. Contributions of different charge states to the distribution $\mathrm{N}^{5+}$ after Auger relaxation are denoted by different shadings.

More detailed information can be extracted when the decay of the multiply excited state by photon or electron emission could be measured in coincidence with the scattering angle. Figure 16 shows the angular distributions integrated over all distances of closest approach for HCI's for different charge states. At the exit of the capillary, the charge states are clearly separated with respect to the scattering angle. The larger the scattering angle the lower the charge state. The total number of events of $i$ th charge state, as described above, is related to the area of the annulus between the radius $\rho_{c}(i)$ and $\rho_{c}(i-1)$. However, this clear separation breaks down at macroscopic distances as a result of relaxation. The number of effective single-capture events is strongly enhanced compared with other charge states. Nevertheless, these angular distributions can be used to identify different phases of hollow ion formation. At macroscopic distances, we can distinguish three regions for the single-capture $\mathrm{N}^{5+}$ states: (1) Within the scattering angle between 1.12 and 1.17 degrees, the ion that preserved its originally captured one electron from the surface. These trajectories never came closer to the surface than $d_{c}\left(\mathrm{~N}^{4+}\right)$. This corresponds to the first stage of hollow-atom formation: the capture of single electrons into a Rydberg state. (2) From scattering angle 1.17 to 1.21 degrees, only $\mathrm{N}^{5+}$ ions can be seen in the angular distributions. But in this region, the original one-electron capture states are contaminated by the one-electron capture states generated by relaxation. The second region represents predominantly the second stage of hollow-atom formation with double capture followed by a single Auger relaxation. (3) While in the previous two regions $\mathrm{N}^{5+}$ is the only charge state observed asymptotically, at scattering angles larger than $1.21^{\circ}$ the asymptotically observed charge state $\mathrm{N}^{5+}$ is mixed with other charge states. Lower charge states can be seen in the angular distributions. Because of the overlap between the angular distributions of $\mathrm{N}^{5+}$ and $\mathrm{N}^{4+}$ at exit, some coincident information would be required to completely disentangle the stages. For example, detecting an electron (of any energy) in coincidence with a projectile in the second angular sector (without a charge-state analysis) would permit to unambiguously identify the second stage of hollow atom formation. For angles larger than $1.25^{\circ}, \mathrm{N}^{5+}$ originates from the multiple Auger decay of an (almost) neutral hollow atom, primarily from $\mathrm{N}^{2+}$ and $\mathrm{N}^{+}$. Clearly, extending this analysis to later stages gets progressively more involved. Depending on the number and energy of ejected electrons or photons, more detailed information may be extracted.

We note that the angular distributions displayed in Figs. 15 and 16 were calculated under the assumption that the image acceleration is given by Eqs. (29) and (30) for a metallic Ni surface. This is equivalent to the assumption that the interior surface of the capillary is atomically clean and can be characterized by the dielectric function for Ni. However, if the image acceleration is suppressed due to surface contamination (e.g., by hydrocarbons) the angular distribution is drastically changed. As a test, we completely neglected the image acceleration. In this case the angular distributions for all charge states are centered around zero degrees.

\section{SUMMARY AND OUTLOOK}

In this paper, we have presented classical trajectory calculations for HCI's passing through a microcapillary target including the simulation of the neutralization and relaxation cascade. The simulation treats the ionic and electronic degrees of freedom simultaneously. We find the fraction of transmitted projectiles that are partially neutralized in good agreement with recent experiments. We have presented a theoretical model yielding an almost flat charge-state distribu- 
tion that is unique to this process and has not been observed before in either ion-atom, ion-solid, or ion-surface collisions. Moreover, the charge-state dependent correlation between scattering angle and distance of closest approach predicts that angular-resolved charge-state distributions may provide direct information on the evolution of the charge cloud of a hollow atom at large distances from the surface. Although the separation of charge states in the angular distributions at large distances from the surface is partially destroyed because of the relaxation, this system offers the promise of disentangling some of the stages of hollow-atom formation. Since final charge-state depends on (1) the initial electron distributions among different shells, (2) the speed of the relaxation processes, and (3) the image acceleration, comparison between experimental and theoretical data also provides a critical test for theory.
We close by pointing out that the description developed in this paper can be also applied to the emerging field of interaction of ions with nanotubes [44]. One important difference between these systems and the microcapillary is the drastically different ratios between critical distance for electronic processes to tube (or track) diameter. Also, the aspect ratios (diameter to length) will be different. Otherwise, most of the key elements of the description would apply. In particular, local tests of the dielectric response of nanotubes, i.e., its interior would become possible. Research along those lines is planned.

\section{ACKNOWLEDGMENT}

The work was supported by the Austrian Fonds zur Förderung der wissenschaftlichen Forschung (P12470-TPH).
[1] P. Varga, Comments At. Mol. Phys. 23, 111 (1989).

[2] J. P. Briand, L. de Billy, P. Charles, S. Essabaa, P. Briand, R. Geller, J. P. Desclaux, S. Bliman, and C. Ristori, Phys. Rev. Lett. 65, 159 (1990).

[3] J. Burgdörfer, P. Lerner, and F. W. Meyer, Phys. Rev. A 44, 5674 (1991).

[4] J. N. Bardsley and B. M. Penetrante, Comments At. Mol. Phys. 27, 43 (1991).

[5] F. W. Meyer, S. H. Overbury, C. C. Havener, P. A. Zeijlmans van Emmichoven, J. Burgdörfer, and D. M. Zehner, Phys. Rev. A 44, 7214 (1991).

[6] H. Winter, Europhys. Lett. 18, 207 (1992).

[7] J. Burgdörfer, in Fundamental Processes and Applications of Atoms and Ions, edited by C. D. Lin (World Scientific, Singapore, 1993), pp. 517-614.

[8] H. Winter, C. Auth, R. Schuch, and E. Beebe, Phys. Rev. Lett. 71, 1939 (1993).

[9] F. W. Meyer, L. Folkerts, I. G. Hughes, S. H. Overbury, D. M. Zehner, P. A. Zeijlmans van Emmichoven, and J. Burgdörfer, Phys. Rev. A 48, 4479 (1993).

[10] J. Das and R. Morgenstern, Comments At. Mol. Phys. 29, 205 (1993).

[11] L. Folkerts, S. Schippers, D. M. Zehner, and F. W. Meyer, Phys. Rev. Lett. 74, 2204 (1995).

[12] A. Arnau, R. Köhrbrück, M. Grether, A. Spieler, and N. Stolterfoht, Phys. Rev. A 51, R3399 (1995).

[13] J. P. Briand, G. Giardino, G. Borsoni, M. Froment, M. Eddrief, C. Sébenne, S. Bardin, D. Schneider, J. Jin, H. Khemliche, Z. Xie, and M. Prior, Phys. Rev. A 54, 4136 (1996).

[14] C. Lemell, H. P. Winter, F. Aumayr, J. Burgdörfer, and F. W. Meyer, Phys. Rev. A 53, 880 (1996).

[15] Q. Yan, D. M. Zehner, F. W. Meyer, and S. Schippers, Phys. Rev. A 54, 641 (1996).

[16] A. Arnau, F. Aumayr, P. M. Echenique, M. Grether, W. Heiland, J. Limburg, R. Morgenstern, P. Roncin, S. Schippers, R. Schuch, N. Stolterfoht, P. Varga, T. J. M. Zouros, and H. P. Winter, Surf. Sci. Rep. 27, 113 (1997).

[17] Q. Yan, J. Burgdörfer, and F. W. Meyer, Phys. Rev. B 56, 1589 (1997).
[18] W. Huang, H. Lebius, R. Schuch, M. Grether, and N. Stolterfoht, Phys. Rev. A 56, 3777 (1997).

[19] L. Hägg, C. O. Reinhold, and J. Burgdörfer, Phys. Rev. A 55, 2097 (1997).

[20] C. Lemell, J. Stöckl, J. Burgdörfer, G. Betz, H. P. Winter, and F. Aumayr, Phys. Rev. Lett. 81, 1965 (1998).

[21] J. J. Ducrée, F. Casali, and U. Thumm, Phys. Rev. A 57, 338 (1998).

[22] H. P. Winter and F. Aumayr, J. Phys. B 32, R39 (1999).

[23] See for example: Proceedings of the 8th International Conference on the Physics of Highly Charged Ions (1996) [Phys. Scr., $\mathrm{T} 73$ (1997)]; Proceedings of the 9th International Conference on the Physics of Highly Charged Ions (1998) [ibid. 80 (1999)].

[24] R. Köhrbrück, M. Grether, A. Spieler, N. Stolterfoht, R. Page, A. Saal, and J. Bleck-Neuhaus, Phys. Rev. A 50, 1429 (1994).

[25] J. Limburg, S. Schippers, I. Hughes, R. Hoekstra, R. Morgenstern, S. Hustedt, N. Hatke, and W. Heiland, Phys. Rev. A 51, 3873 (1995).

[26] N. Stolterfoht, A. Arnau, M. Grether, R. Köhrbrück, A. Spieler, R. Page, A. Saal, J. Thomaschewski, and J. Bleck-Neuhaus, Phys. Rev. A 52, 445 (1995).

[27] R. Page, A. Saal, J. Thomaschewski, L. Aberle, J. BleckNeuhaus, R. Köhrbrück, M. Grether, and N. Stolterfoht, Phys. Rev. A 52, 1344 (1995).

[28] F. W. Meyer, S. H. Overbury, C. C. Havener, P. A. Zeijlmans van Emmichoven, and D. M. Zehner, Phys. Rev. Lett. 67, 723 (1991).

[29] F. W. Meyer, C. C. Havener, and P. A. Zeijlmans van Emmichoven, Phys. Rev. A 48, 4476 (1993).

[30] H. Masuda and K. Fukuda, Science 268, 1466 (1995).

[31] H. Masuda and M. Satoh, Jpn. J. Appl. Phys., Part 2 35, L126 (1996).

[32] Y. Yamazaki, S. Ninomiya, F. Koike, H. Masuda, T. Azuma, K. Komaki, K. Kuroki, and M. Sekiguchi, J. Phys. Soc. Jpn. 65, 1199 (1996).

[33] Y. Yamazaki, Phys. Scr., T 73, 293 (1997).

[34] S. Ninomiya, Y. Yamazaki, F. Koike, H. Masuda, T. Azuma, K. Komaki, K. Kuroki, and M. Sekiguchi, Phys. Scr., T 73, 316 (1997). 
[35] S. Ninomiya, Y. Yamazaki, F. Koike, H. Masuda, T. Azuma, K. Komaki, K. Kuroki, and M. Sekiguchi, Phys. Rev. Lett. 78, 4557 (1997).

[36] Y. Yamazaki, Invited papers of XXth International Conference on the Physics of Electronic and Atomic Collisions, Vienna, 1997, in Photonic, Electronic and Atomic Collisions, edited by F. Aumayr and H. P. Winter (World Scientific, Singapore, 1998), p. 693.

[37] K. Tőkési, L. Wirtz, C. Lemell, and J. Burgdörfer, Phys. Rev. A 61, 020901(R) (2000).

[38] K. Tókési, L. Wirtz, C. Lemell, and J. Burgdörfer, Nucl. Instrum. Methods Phys. Res. B 164-165, 504 (2000).

[39] K. Tőkési and J. Burgdörfer, Surf. Sci. 454-456, 1038 (2000).

[40] R. H. Ritchie and A. L. Marusak, Surf. Sci. 4, 234 (1966).

[41] D. Chan and P. Richmond, Surf. Sci. 39, 437 (1973).

[42] J. D. Jackson, Classical Electrodynamics, 2nd ed. (Wiley, New York, 1975).

[43] K. Tökési, L. Wirtz, and J. Burgdörfer, Nucl. Instrum. Methods Phys. Res. B 154, 307 (1999).

[44] C. R. Vane and S. Datz (private communications).

[45] R. H. Ritchie and A. Howie, Philos. Mag. 36, 463 (1977).

[46] J. C. Ashley, J. J. Cowan, R. H. Ritchie, V. E. Anderson, and J. Hoelzl, Thin Solid Films 60, 361 (1979).

[47] B. Gervais, C. O. Reinhold, and J. Burgdöfer, Phys. Rev. A 53, 3189 (1996).

[48] D. W. Lynch and W. R. Hunter, in Handbook of Optical Con- stant of Solids, edited by E. D. Palik (Academic Press, London, 1985).

[49] P. Nozieres and D. Pines, The Theory of Quantum Liquids (Addision-Wesley, Reading, MA, 1990).

[50] D. R. Penn, Phys. Rev. B 35, 482 (1987).

[51] B. I. Lundqivst, Phys. Kondens. Mater. 6, 206 (1967).

[52] W. R. Smythe, Static and Dynamic Electricity, 2nd ed. (McGraw-Hill, New York, 1950).

[53] M. Grether, A. Spieler, R. Köhrbrück, and N. Stolterfoht, Phys. Rev. A 52, 426 (1995).

[54] N. Vaeck and J. E. Hansen, J. Phys. B 28, 3523 (1995).

[55] E. J. McGuire, Phys. Rev. A 3, 587 (1971).

[56] D. L. Walters and C. P. Bhalla, Phys. Rev. A 3, 1919 (1971).

[57] C. P. Bhalla and M. Hein, Phys. Rev. Lett. 30, 39 (1973).

[58] R. Díez Muiño, A. Salin, N. Stolterfoht, A. Arnau, and P. M. Echenique, Phys. Rev. A 57, 1126 (1998).

[59] J. C. Slater, Phys. Rev. 36, 57 (1930).

[60] H. D. Hagstrum, Phys. Rev. 96, 325 (1954).

[61] H. D. Hagstrum, Phys. Rev. 96, 336 (1954).

[62] R. D. Cowan, The Theory of Atomic Structure and Spectra (University of California Press, Berkeley, 1981).

[63] P. Zeijmans Van Emmichoven, C. Havener, and F. Meyer, Phys. Rev. A 43, 1405 (1991).

[64] H. A. Bethe and E. E. Salpeter, Quantum Mechanics of Oneand Two-Electron Atoms (Plenum, New York, 1977).

[65] J. Burgdörfer and F. Meyer, Phys. Rev. A 47, R20 (1993).

[66] Y. Yamazaki (private communications). 